\title{
Biotransformation of $\quad 6: 2$ fluorotelomer alcohol by the whole soybean (Glycine max L. Merrill) seedlings
}

Hongna Zhang ${ }^{1,2}$, Bei Wen ${ }^{1, *}$, Honglin Huang ${ }^{1}$, Zongwei $\mathrm{Cai}^{2}$, Shuzhen Zhang ${ }^{1}$

1 State Key Laboratory of Environmental Chemistry and Ecotoxicology, Research Center for Eco-Environmental Sciences, Chinese Academy of Sciences, Beijing 100085, China

2 State Key Laboratory of Environmental and Biological Analysis, Department of Chemistry, Hong Kong Baptist University, Hong Kong 999077, China

Fluorotelomer alcohols (FTOHs) are important precursors of perfluorocarboxylic acids (PFCAs) in environmental media and biota. With the growing application of $6: 2 \mathrm{FTOH}$ $\left[\mathrm{F}(\mathrm{CF} 2)_{6} \mathrm{CH} 2 \mathrm{CH} 2 \mathrm{OH}\right]$ in product formulation, it is becoming increasingly urgent to investigate the biotransformation of $6: 2$ FTOH in different biological species. In this study, young soybean plants (Glycine max L. Merrill) were hydroponically exposed to 6:2 FTOH for 12-144 h. During the exposure, 6:2 FTCA and 6:2 FTUCA were the primary intermediates in soybean tissues. At the end of exposure, 5:3 FTCA (5.08 mol\%), PFHxA (2.34 mol\%) and PFPeA (0.58 mol\%) were three main phase I metabolites in soybean-solution system. 5:3 FTCA was predominant in soybean roots and stems, while PFHxA was the most abundant metabolite in leaves. PFBA and 4:3 FTCA were also generated in the culture solution, most-likely from the transformation of 5:3 FTCA by root-associated microbes. Moreover, phase II metabolites of 6:2 FTOH were identified and monitored in soybean tissues. Alcohol dehydrogenase, aldehyde dehydrogenase and glutathione S-transferase were found to participate in 6:2 FTOH metabolism. This study for the first time provides evidences for the transformation pathways of $6: 2 \mathrm{FTOH}$ in plant.

Acknowledgments We thank the National Natural Science Foundation of China (Projects 41877479, 21537005 , 21577155 and 21806134) for financial support. 